\title{
Reliable Multicast Sessions Provisioning in Sparse Light-Splitting DWDM Networks using P-Cycles
}

\author{
Ahmed Frikha*, Samer Lahoud*, Bernard Cousin*, and Miklos Molnar ${ }^{\dagger}$ \\ ${ }^{*}$ University of Rennes 1, IRISA, France \\ Email:\{afrikha,slahoud,bcousin\}@irisa.fr \\ ${ }^{\dagger}$ University of Montpellier 2, LIRMM, France \\ Email: molnar@lirmm.fr
}

\begin{abstract}
In this paper, we study the node and link protection using p-cycles for dynamic multicast sessions in alloptical DWDM networks. First, we propose a new concept for protecting nodes of light-trees under the sparse light-splitting and wavelength continuity constraints. Then, we integrate our concept in a novel algorithm, named node and link protecting candidate p-cycles based algorithm with sparse light-splitting constraints (NPCC-SSC). Our algorithm enables both node and link failure recovery in dynamic multicast traffic. Extensive simulations show that the NPCC-SSC algorithm achieves the best resource utilization, and outperforms the existing approaches in terms of blocking probability and computational time.
\end{abstract}

\section{INTRODUCTION}

All-optical DWDM network provides a high bandwidth as it allows hundreds of wavelengths to be multiplexed onto a single fiber. Furthermore, all-optical networks allow multicasting to be performed at the optical layer through light-splitting [1]. The light-splitting or multicast capable (MC) nodes are, however, expensive and complex to fabricate. Therefore the MC nodes are sparsely configured in practical DWDM networks [2]. The rest of the nodes in the network are multicast incapable (MI). In this paper, we designate by sparse lightsplitting network, a network where a small percentage of nodes are $\mathrm{MC}$ and are well placed in the network to ensure efficient multicasting [2]. We also assume that the sparse light-splitting networks have no wavelength convertors.

Maintaining network survivability is very important since a single link or node failure would affect a large number of communication sessions. In multicast communications, this impact is more severe as a link or node may carry traffic for multiple destinations. Hence, protecting multicast sessions in all-optical DWDM networks is a crucial task. The p-cycle protection approach, introduced by W.D. Grover in [3] for unicast traffic, ensures a fast restoration time since p-cycles are pre-cross-connected. When a link fails, the restoration process is handled by the end nodes of the failed link. Moreover, the pcycle protection approach provides a high capacity efficiency as it allows both on-cycle and straddling links to be protected by the p-cycle. In 2007, F. Zhang and W.D. Zhong showed in [4] that applying p-cycle protection concept for multicast traffic leads to a blocking probability very low compared with that of the OPP-SDP algorithm [5].

Up to now, most of existing researches in optical multicast traffic focus on link failure recovery and rarely on node failure recovery. Although node failures are less frequent than link failures, node failures may cause the disruption of multiple communications, especially when the failed node carries traffic for multiple destinations. In 2009, F. Zhang and W.D. Zhong proposed a heuristic algorithm for combined node and link failure recovery, named ESHT [6]. The ESHT algorithm is based on p-cycle concept. Then, in [7], they proposed an enhanced version of ESHT, named ESHN. The ESHN algorithm achieves the best resource utilization and has the lowest blocking probability among the OPP-SDP algorithm [5] and the ESHT algorithm in dynamic multicast traffic. In [8], we proposed two new algorithms for link and node protection using p-cycles, named NPC and NPCC. The NPC algorithm achieves the lowest blocking probability, but has the highest computational time among the NPCC and ESHN algorithms. The NPCC algorithm outperforms the ESHN algorithm in terms of resource utilization efficiency and computational time.

All of these algorithms are designed for optical DWDM networks where every node is equipped with wavelength converter and light-splitter. They are not applicable in sparse light-splitting networks as they do not take into consideration the sparse light-splitting and wavelength continuity constraints when computing a new p-cycle. The wavelength continuity constraint means that the same wavelength must be used in all the links of the light-tree as well as its corresponding protection structures, so that there is no need to wavelength converters at each node. The sparse light-splitting constraint means that when routing and protecting a light-tree, nodes that are not equipped with light-splitter (MI nodes) are taken into consideration, and it is not possible to split the wavelength in these nodes. For example, an MI node must not be a branching node in the light-tree. Works in [9]-[10] proposed two multicast protection algorithms for sparse light-splitting WDM networks. These algorithms satisfy the sparse lightsplitting and wavelength continuity constraints when computing the protection structures for the light-trees. These solutions implement the path-based protection approach, and compute for each destination node in the light-tree a backup path that can share some links with the active path. The path-based protection approach, however, do not provide a fast restoration time as the restoration is handled by the extremity nodes of the path. 
In this paper, we study the link and node protection using $\mathrm{p}$ cycles for dynamic multicast sessions in sparse light-splitting DWDM networks. We propose a novel concept for node protection under the sparse light-splitting and wavelength continuity constraints. Then, we propose a novel algorithm that deploys our concept for the node protection, named node and link protecting candidate p-cycles based algorithm with sparse light-splitting constraints (NPCC-SSC). This algorithm relies on a set of well selected candidate p-cycles instead of using all the network p-cycle set. This will reduce the computational time of setting up a multicast session to deal with the dynamic traffic. We also adapt the ESHN algorithm to deal with the sparse light-splitting and wavelength continuity constraints. Extensive simulations show that the NPCC-SSC algorithm achieves the best resource utilization, and outperforms the ESHN algorithm in terms of blocking probability and computational time.

The rest of this paper is organized as follows. In section II, we present our concept for protecting nodes using pcycles under sparse light-splitting constraints. In section III, we present our novel algorithm for combined node and link failure recovery with sparse light-splitting constraints. Performed simulations and numerical results are presented in section IV. The conclusions are given in section V.

\section{NODE PROTECTION CONCEPT WITH SPARSE LIGHT-SPLITTING CONSTRAINTS}

We assume that a small percentage of networks nodes are MC. The rest are MI. MI nodes can be classified into two types: Tap or Continue (ToC) MI nodes and Tap and Continue (TaC) MI nodes [11]. In this work, we assume that all MI nodes in the network are Tape and Continue (TaC) capable. Like that if an MI node is a destination and an intermediate node in the light-tree, it can tape a small fraction of the input signal for the local router and switches the remaining power to any one of the other outputs. We also assume that there is no wavelength convertors in the network nodes. Therefore, the same wavelength must be used in all the links of a light-tree and its protection p-cycles to satisfy the wavelength continuity constraint.

Now, let us introduce some notations before presenting our concept. Let $T$ be a multicast light-tree to be protected, $N_{f}$ be an intermediate node in $T$, and $D=\left\{d_{1}, d_{2}, . ., d_{i}\right\}$ be the set of destinations of $T$ that are affected when a failure occurs on the node $N_{f}$.

A p-cycle $C$ of the network can protect the node $N_{f}$ if and only if it exists a protection segment $\left[N_{a}, N_{e}\right] \in C$ such that:

1) $N_{a}$ is the node which activates $C$ when $N_{f}$ fails. $N_{a}$ is not affected by the failure of $N_{f}$ and:

- $N_{a}$ is an MC node.

- Or $N_{a}$ is a leaf destination MI node.

- Or $N_{a}$ is an MI node and every destination $d_{i}$, descendant of $N_{a}$ and $d_{i} \notin D$, is covered by the protection segment $\left[N_{a}, N_{e}\right]$. As $N_{a}$ cannot split the signal to these destinations, they cannot receive the signal through $N_{a}$ any more. These destinations are denoted by the rerouting affected destinations $D_{r e}$, and must be added to the set of affected destination $D$ to be covered by $\left[N_{a}, N_{e}\right]: D \leftarrow D \cup D_{r e}$.

2) $\forall d_{j} \in D, \exists N_{j} \in\left[N_{a}, N_{e}\right]$ and:

- $N_{j}=d_{j}$.

- Or $N_{j}=N_{e}$.

- Or $N_{j}$ is an MC node and $N_{f} \notin\left[N_{j}, d_{j}\right]$.

3) $N_{f} \notin\left[N_{a}, N_{e}\right]$.

The node $N_{a}$ must inject the multicast traffic in the p-cycle upon the failure of $N_{f}$. Therefore, this node must not be affected by the failure of $N_{f}$, i.e. $N_{a}$ continues to receive the multicast traffic even if a failure occurs on node $N_{f}$. If $N_{a}$ is an MC node, it splits the input light signal into two light signals: the first signal is injected in $C$ and the second is forwarded to the next node of $N_{a}$ in the light-tree. If $N_{a}$ is a leaf destination node, it tapes a fraction of the input light signal and injects the rest in $C$. Otherwise, $N_{a}$ can reroute the input light signal: it injects the signal in $C$ instead of the next node of $N_{a}$ in the light-tree. However, in this case some destinations can be affected by the rerouting, therefore we add these destinations to the set of the affected destinations $D$ : $D \leftarrow D \cup D_{r e}$. Constraint 2) ensures that each destination $d_{j}$ in $D$ (affected by the failure of $N_{f}$ or by an eventual rerouting on the node $N_{a}$ ) continues to receive the multicast traffic through the protection segment $\left[N_{a}, N_{e}\right]$. The protection segment can route the multicast traffic to $d_{j}$ through an intermediate MC node $N_{j}$, where $N_{f}$ must not be in the segment $\left[N_{j}, d_{j}\right]$ of the light-tree. In this cas, node $N_{j}$ must split the input light signal into two light signals: the first signal is forwarded to the next node of $N_{j}$ in $C$ and the second is forwarded to the next node of $N_{j}$ in $T$. If $N_{j}$ is the extremity of the protection segment $\left(N_{j}=N_{e}\right)$, it can be MI as it will not forward the light signal to the next node in $C$. The last possible case is that the protection segment $\left[N_{a}, N_{e}\right]$ routes directly the traffic to $d_{j}\left(N_{j}=d_{j}\right)$. Constraint 3$)$ ensures that the protection segment $\left[N_{a}, N_{e}\right]$ does not traverse the failed node $N_{f}$.

Fig. 1, Fig. 2, and Fig. 3 illustrate some examples of pcycles that can protect the node $N_{f}$ using our concept. In Fig. 1, the node $N_{a}$, which activates the p-cycle, is a leaf destination node so it can tape a small fraction of the input signal for the local router and switches the remaining power to the p-cycle. In Fig. 2, the node $N_{a}$ is MI and is not a leaf node, therefore it reroutes the traffic to the p-cycle. In this case, however, there is a destination node affected by the rerouting. This destination will continue to receive the traffic through the protection segment $\left[N_{a}, N_{e}\right]$. In Fig. 3, the node $N_{a}$ is $\mathrm{MC}$, so it splits the input light signal into two light signals: one signal is forwaded to the next node in $\left[N_{a}, N_{e}\right]$ and the second signal is forwarded to the descendant of $N_{a}$ in $T$.

\section{THE PROPOSED ALGORITHMS}

In this section, first we present our novel algorithm NPCCSSC for combined node and link failure recovery under the sparse light-splitting and wavelength continuity constraints. 

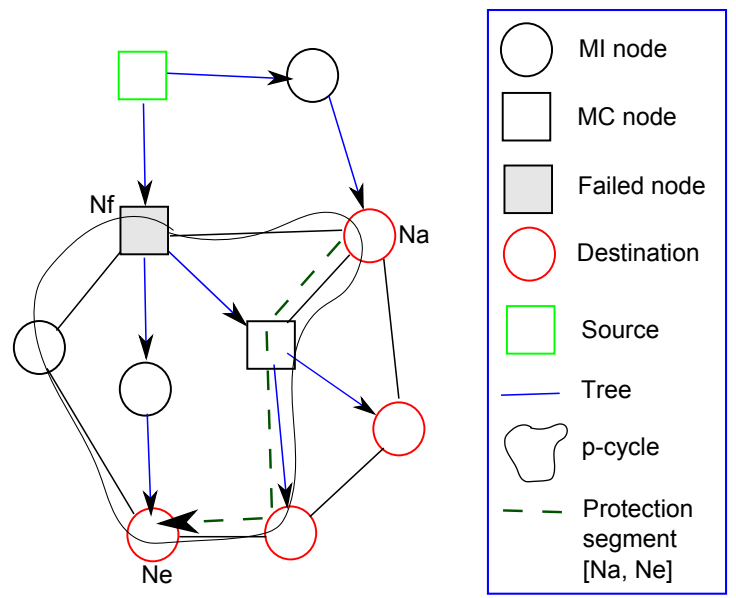

Fig. 1. $\quad N_{a}$ is a leaf destination MI node
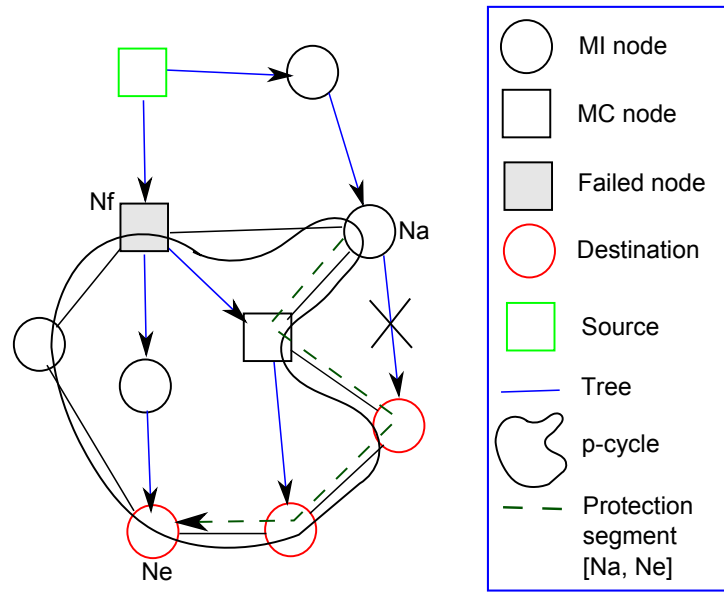

Fig. 2. $\quad N_{a}$ is an intermediate MI node
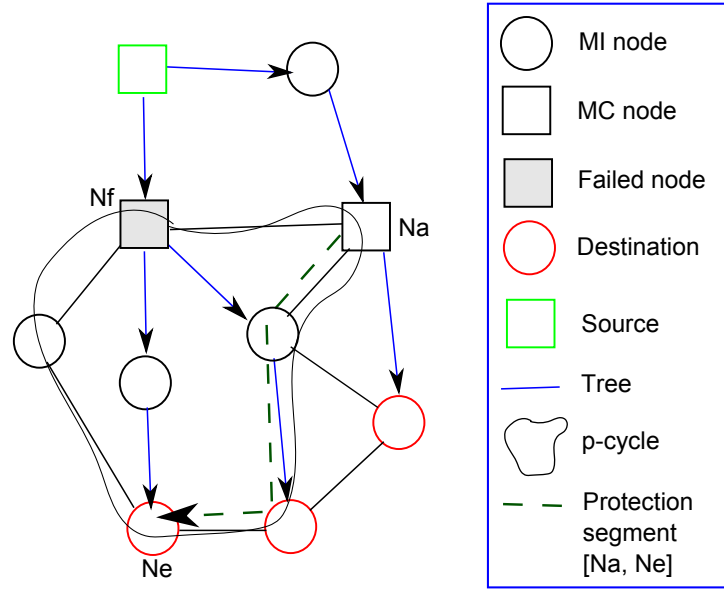

Fig. 3. $N_{a}$ is an MC node

Our algorithm deploys the aforementioned concept for node protection. Second, we propose an adaptation for the ESHN algorithm, named ESHN improved, to deal with the sparse light-splitting constrained networks.

\section{A. The NPCC-SSC algorithm}

Fig. 4 presents the flow chart of the NPCC-SSC algorithm. Upon the reception of a new multicast request, our algorithm attempts to route and protect the multicast light-tree using the same wavelength in all the links. The computation starts with the first wavelength $w_{1}(i=1$ in the flow chart), the algorithm tries to route the request using the wavelength $w_{1}$, if a multicast light-tree is found, our algorithm tries to protect all the link capacity and all the node transit capacity of the computed light-tree using $w_{i}$ to preserve the wavelength continuity constraint. If the request cannot be routed and protected using $w_{1}$, the algorithm tries to route and protect the multicast request using the next wavelength $w_{2}(i=i+1)$. This process is iterated with each wavelength $w_{i}$ until a multicast light-tree is routed and protected using one single wavelength $w_{i}$. Otherwise, the request is rejected. Note that the requests are routed using the algorithm described in [12].

To detail the operations performed by the NPCC-SSC algorithm when protecting a multicast light-tree, we suppose that for a given wavelength $w_{i}$, the algorithm finds a multicast light-tree, denoted by $T$. Let $L$ denote the unprotected working link capacity of $T$, and $N$ denote the unprotected intermediate node transit capacity of $T$. First of all, the algorithm finds the amount of working link capacity and the amount of node transit capacity that can be protected according to our proposed concept for the node protection, by the existing p-cycles reserved with $w_{i}$ in the network. The amount of working link capacity protected by these p-cycles is subtracted from $L$ and the amount of protected node transit capacity is subtracted from $N$. Note that these existing p-cycles are previously established to protect other light-trees routed with $w_{i}$. If $L \neq \phi$ or $N \neq \phi$, the algorithm computes new p-cycles using $w_{i}$ to protect the remaining unprotected link capacity in $L$ as well as the remaining unprotected node transit capacity in $N$.

To select a new protecting p-cycle, the algorithm uses a new score $S$ to measure the efficiency of the p-cycles in the network. This score takes into consideration the largest amount of unprotected node transit capacity that a p-cycle reserved with $w_{i}$ can protect according to our proposed concept for the node protection. The score $S$ takes also into consideration the largest amount of unprotected working link capacity of the multicast tree that the p-cycle can protect. Let $C_{j}$ be a pcycle in the network. The score $S$ of $C_{j}$ is given by equation (1), where $L C_{j, L}$ is the largest amount of unprotected link capacity in $L$ that $C_{j}$ can protect, $N T C_{j, N}$ is the largest amount of unprotected node transit capacity in $N$ that $C_{j}$ can protect according to our concept, and $\left|C_{j}\right|$ is the spare capacity required for setting up $C_{j} .\left|C_{j}\right|$ is given by the number of links traversed by $C_{j}$.

$$
S\left(C_{j}\right)=\frac{L C_{j, L}+N T C_{j, N}}{\left|C_{j}\right|}
$$

The algorithm calculates the score $S$ of each p-cycle and selects the p-cycle with maximum $S$. The amount of working 
link capacity protected by the selected p-cycle is subtracted from $L$ and the amount of protected node transit capacity is subtracted from $N$. This process is iterated until the amount of working link capacity in $L$ and the amount of node transit capacity in $N$ are protected, i.e. $L=\phi$ and $N=\phi$. The selected p-cycles are configured and the spare capacity of each p-cycle are reserved. Note that the reserved p-cycles may serve to protect next coming multicast requests routed with $w_{i}$. This is why after routing a multicast tree, we compute the amount of working link capacity in $L$ and the amount of node transit capacity in $N$ that can be protected by the existing p-cycles in the network. The reserved capacity of an existing p-cycle in the network is released when the p-cycle does not protect any working link capacity and any node transit capacity.

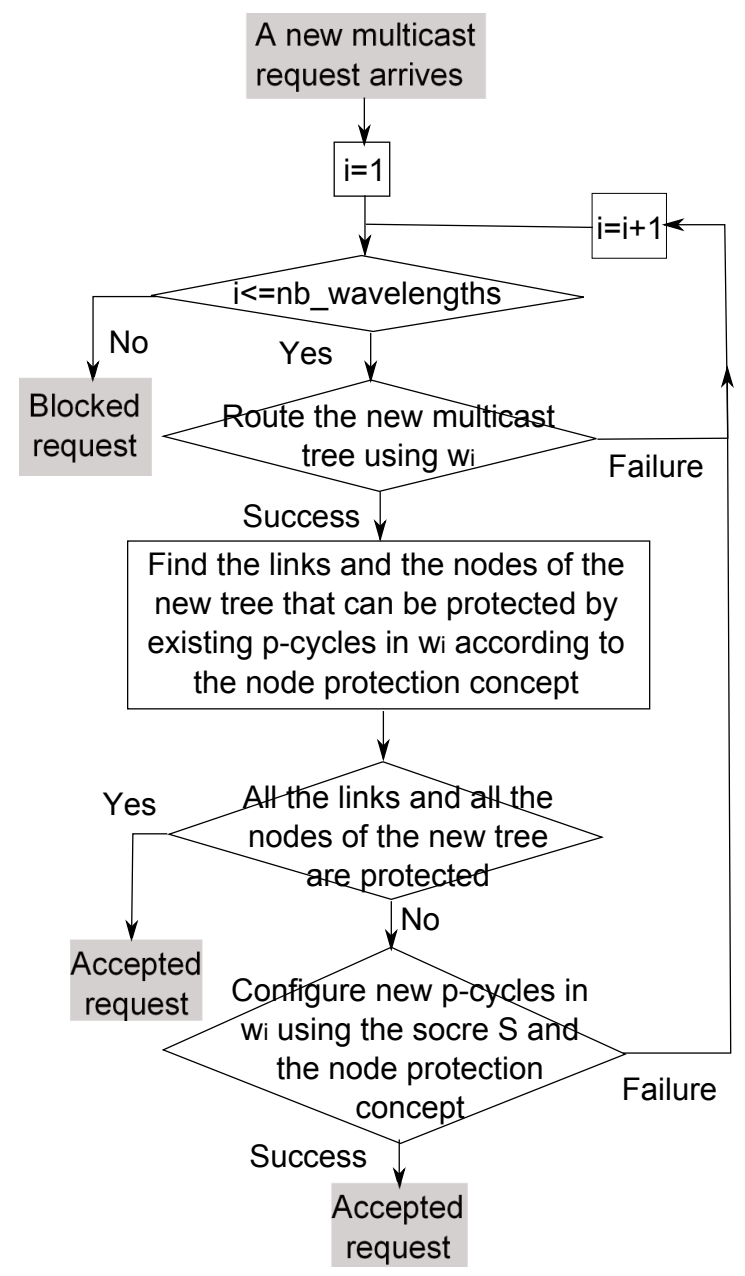

Fig. 4. Flow chart of the NPCC-SSC algorithm for combined link and node failure recovery for dynamic multicast traffic in sparse light-splitting networks

To maintain a reasonable computational time for the algorithm and deal with the dynamic traffic, the NPCC-SSC algorithm relies on a well selected candidate p-cycle set that will be used when searching for a new p-cycle. To select a new p-cycle with maximum $S$, our algorithm do not compute the score $S$ of each p-cycle in the total network p-cycle set, but only the score $S$ of each candidate p-cycles. This will reduce considerably the computational time of the algorithm.
In fact, when the number of p-cycles in the network is high, computing the score $S$ of each p-cycle in the network is a very long task and affects the computational time of the algorithm. Therefore, we select a set of candidate p-cycles to reduce the computational time. To select a candidate p-cycle set, we define a new score, named protection capacity $P C$, for each p-cycle in the network. This score is computed in advance for each p-cycle before routing the requests. The score $P C$ of a p-cycle $C_{j}$, specified by equation (2), is defined as the ratio of the largest amount of link capacity on the network $L C_{j}$ that $C_{j}$ can protect over the sum of spare capacity required by $C_{j}$.

$$
P C\left(C_{j}\right)=\frac{L C_{j}}{\left|C_{j}\right|}
$$

A p-cycle with a high $P C$, is useful as it maximizes the amount of protected capacity while reserving less spare capacity. The $l$ p-cycles with highest $P C$ are selected as candidate p-cycle set, where $l$ is a parameter for the algorithm. The goal of selecting this set is to maximize the capacity that can be protected on the network, and this will help to protect the next coming requests. The NPCC-SSC algorithm uses the $l$ selected p-cycles as a candidate p-cycle set instead of using all p-cycles in the network when computing a new p-cycle.

\section{B. The ESHN improved algorithm}

In the ESHN algorithm, a p-cycle $C$ protecting a node $N_{f}$ has to traverse 1) all one level downstream nodes of the node $N_{f}$ and 2) one of its upstream nodes in the light-tree. As the one level downstream nodes and the upstream nodes of node $N_{f}$ can be MI, finding a p-cycle that satisfies the constraints 1) and 2), and at the same time the sparse lightsplitting constrains is a complex task. The p-cycles selection in the ESHN algorithm is based on the score ES [7], however this procedure does not verify if the p-cycle satisfies the sparse light-splitting constraints. We improve ESHN by taking into consideration the sparse light-splitting constraints in the score $E S$, i.e. $E S(C)=0$ if the p-cycle $C$ does not meet the sparse light-splitting constraints. The ESHN improved algorithm, however, does not allow the protection capacity of a p-cycle to be used efficiently because of the constraints 1) and 2) imposed by its concept for node protection.

\section{Performance Evaluation}

In this section, we evaluate our proposed algorithm NPCCSSC for link and node failure recovery in sparse light-splitting optical DWDM network, by comparison with the ESHN algorithm as well as the ESHN improved algorithm. In our simulation, we assume that the global network traffic is a combination of unicast and multicast traffic. Multicast traffic requests are generated with a probability $R$, and unicast traffic requests are generated with a probability $1-R$. The request arrival follows a Poisson process with an average arrival rate $\lambda$, and the request holding time follows an exponential distribution with an average holding time $\mu$. Hence, the network offered traffic load is given by $\lambda \mu$. The multicast traffic load is given by $R \lambda \mu$, while the unicast traffic load equals $(1-R) \lambda \mu$. 
We run simulations on the following well known and very often used European optical topologies developed within the COST-266 [13] and COST-239 [14] projects:

- The COST-266 core topology [13] contains 16 nodes and 23 links, with an average nodal degree equals 2.88 . The total number of cycles in this topology equals 236 (118 cycles in each direction).

- The COST-239 topology [14] contains 11 nodes and 26 links, with an average nodal degree equals 4.727 . The total number of cycles in this topology equals 5058 (2029 cycles in each direction).

In our study, without lack of generality we assume that each link has two fibers. The two fibers transmit in opposite directions; 16 wavelengths are available on each fiber. The source and the destinations of each multicast session are randomly selected (uniform distribution law). We choose the number of destinations in each multicast request equals 5, which seems to be reasonable as the total number of nodes in the used topologies is lower than 16 nodes. We compare the performance of the algorithms according to the following performance criteria:

- The Blocking Probability $(B P)$ represents the percentage of requests that cannot be routed or protected among the total number of requests.

- The Resource Utilization $(R U)$ represents the percentage of reserved wavelengths in the network among the total number of wavelengths. $R U=\frac{W_{R}}{E * W}$, where $W_{R}$ represents the total number of wavelengths reserved in the network, $E$ represents the number of fiber in the network, and $W$ the number of wavelengths per fiber.

- The average Computational Time $(C T)$ required for routing and protecting a traffic request.

Performance criteria $B P, R U$, and $C T$ are computed according to the traffic load. For each traffic load value, $2.5 \times 10^{6}$ requests are generated. This number of requests is enough to measure $B P, R U$, and $C T$, with a $95 \%$ confidence interval.

First, we consider the blocking probability of the algorithms. Fig. 5 and Fig. 6 illustrate the blocking probability of the algorithms according to the network offered traffic load in the COST-239 topology. In these two figures, the probability that a traffic request is a multicast request $R=1$. The number of candidate p-cycles $l$ equals 200 for the NPCC-SSC algorithm. In Fig. 5, the number of MC nodes equals 6 . The remaining nodes are MI. The MC nodes are placed in the highest degree nodes. In Fig. 6, only 3 nodes are MC and the remaining nodes are MI. As shown in Fig. 5 and Fig. 6, the ESHN algorithm and its improved version have a blocking probability very high compared to our proposed algorithm NPCC-SSC, especially when the number of MC nodes is low. This high blocking probability is due to the great number of requests blocked because no protection p-cycles are found to protect the nodes in the light-tree using the concept of ESHN. The blocking probability of the ESHN improved algorithm is lower than that of the ESHN algorithm but remains high compared to the NPCC-SSC algorithm.

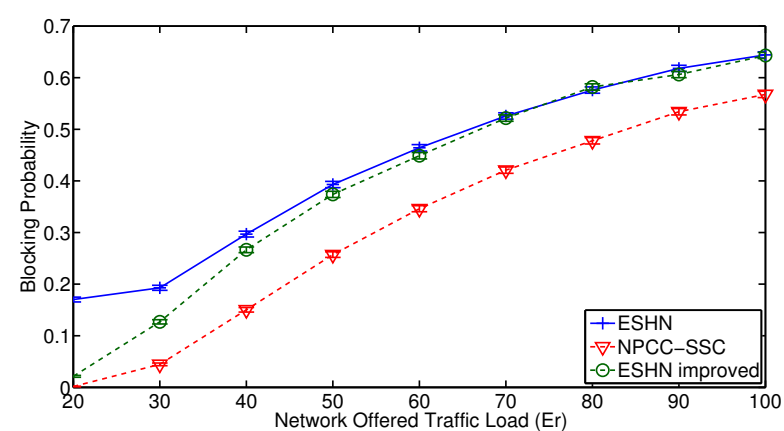

Fig. 5. Comparison of the blocking probability $B P$ in COST-239 network $(\mathrm{R}=1, \mathrm{l}=200, \mathrm{MC}=6)$

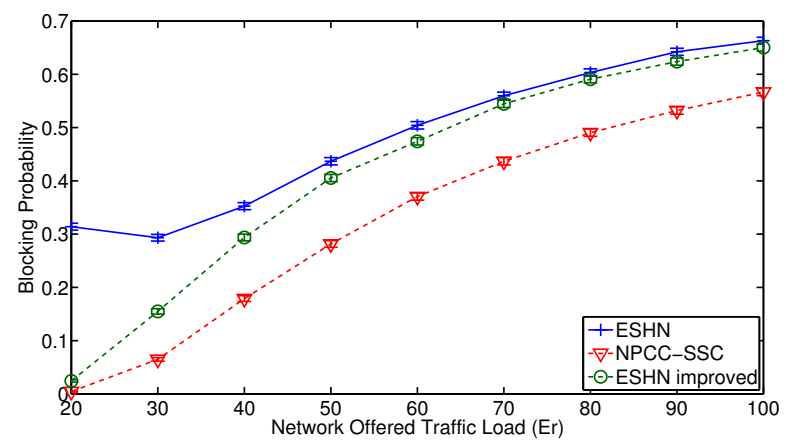

Fig. 6. Comparison of the blocking probability $B P$ in COST-239 network $(\mathrm{R}=1, \mathrm{l}=200, \mathrm{MC}=3)$.

For the COST-266 topology, the number of MC nodes equals 8 and the probability that a traffic request is a multicast request $R=0.23$. As the number of cycles in this topology is low, we consider all the network cycle set as a candidate p-cycles for the NPCC-SSC algorithm $(l=236)$. Fig. 7 illustrates the blocking probability of the algorithms according to the network offered traffic load in the COST-266 topology. The blocking probability of the algorithms is high in general in this topology as the average nodal degree is very low (2.88). However, the blocking probability of the NPCC-SSC algorithm remains always lower than that of the ESHN algorithm and its improved version. Note that simulations with lower numbers of MC nodes and higher values of $R$ are also performed for the COST-266 topology. However, we did not present the results in this paper because of the space limitation. However, we can confirm that the blocking probability of our algorithm is slightly lower than that of the ESHN and the ESHN improved algorithm when $R=0.23$, and is very low compared to these algorithms when $R=1$.

Now, let us focus on the average computational time for setting up a multicast request. Fig. 8 illustrates the average computational time $C T$ for each algorithm according to the network offered traffic load. As expected, the NPCC-SSC algorithm is the most rapid among the ESHN and the ESHN improved algorithms. The $C T$ of NPCC-SSC is always lower than $0.09 \mathrm{~s}$. This is due to the low number of candidate $\mathrm{p}$ cycles $(l=200)$. As shown in Fig. 8, the computational time $C T$ of the ESHN and the ESHN improved algorithms decreases with the offered traffic load augmentation. In fact, the 


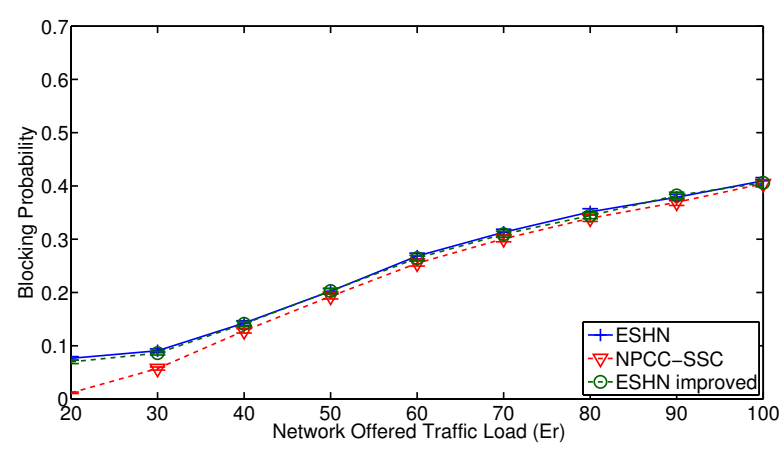

Fig. 7. Comparison of the blocking probability $B P$ in COST-266 network $(\mathrm{R}=0.23, \mathrm{l}=236, \mathrm{MC}=8)$.

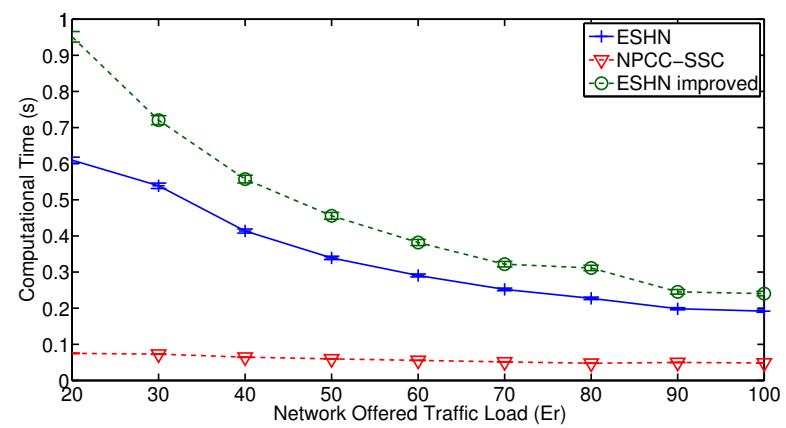

Fig. 8. Comparison of the average computational time $C T$ for setting up a multicast request in COST-239 network $(\mathrm{R}=1, \mathrm{l}=200, \mathrm{MC}=6)$.

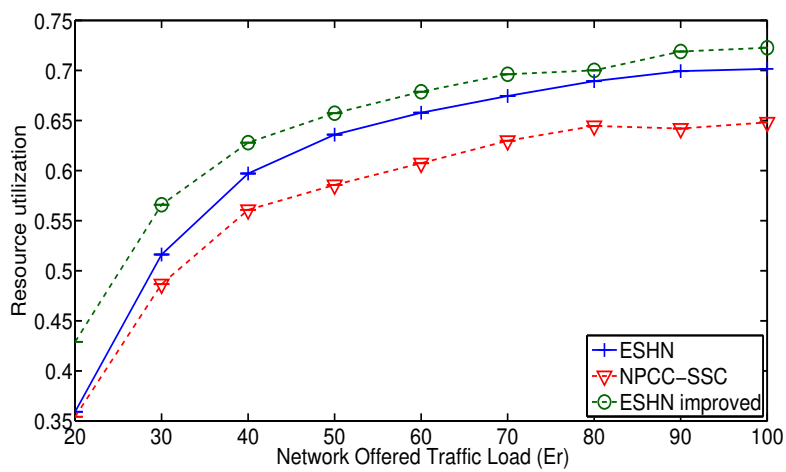

Fig. 9. Comparison of the resource utilization $R U$ in COST-239 network $(\mathrm{R}=1, \mathrm{l}=200, \mathrm{MC}=6)$.

number of the existing p-cycles in the network is higher when the traffic load increases, and the probability of protecting the multicast request using the existing p-cycles increases and this reduces the computational time.

Fig. 9 shows the resource utilization of the algorithms. This figure shows that the $R U$ of the NPCC-SSC algorithm is lower than that of the ESHN and the ESHN improved algorithms. The percentage of wavelengths reserved on each link by the NPCC-SSC algorithm is the lowest. In fact, our concept for the node protection with light-splitting constraints allows the protection capacity of p-cycles to be used efficiently and hence reserving less spare capacity. This will reduce the resource utilization as well as the blocking probability of the algorithm. We did not present the $C T$ and the $R U$ of the algorithms in the COST-266 topology because of the space limitation.

\section{CONClusion}

In this paper, we proposed a novel concept for node protection for light-trees using p-cycles in sparse light-splitting DWDM networks. Our novel concept relaxes the constraints imposed by the existing approaches for protecting nodes of light-trees and deals with the sparse light-splitting and wavelength continuity constraints. This relaxation allows the protection capacity provided by a p-cycle to be used efficiently and therefore achieving good resource utilization. We also proposed a novel algorithm, named NPCC-SSC, for link and node failure recovery of dynamic multicast sessions in sparse light-splitting DWDM network. This algorithm deploys the proposed concept for the node protection. We evaluate our proposed algorithm NPCC-SSC, by comparison with the ESHN algorithm as well as an improved version of ESHN. Extensive simulations showed that the NPCC-SSC algorithm achieves the lowest blocking probability and outperforms the ESHN algorithm and its improved version in terms of resource utilization efficiency and computational time.

\section{REFERENCES}

[1] J.J.V. Olmos, M. Tokushima, and K.I. Kitayama, Photonic Add-Drop Filter Based on Integrated Photonic Crystal Structures, in IEEE Journal of Selected Topics in Quantum Electronics, vol. 16, no. 1, pp. 332-337, 2010.

[2] S. Chen, T.H. Cheng, G.S Poo, Placement of wavelength converters and light splitters in a WDM network using the generic graph model, in Computer Communications, vol. 33, no. 7, pp. 868-883, 2010.

[3] W. D. Grover and D. Stamatelakis, Cycle-oriented distributed preconfiguration: ring-like speed with mesh-like capacity for self-planning network restoration, in proceedings of IEEE ICC, 1998.

[4] F. Zhang and W. D. Zhong, Applying p-cycles in dynamic provisioning of survivable multicast sessions in optical WDM networks, in proceedings Optical Fiber Communications Conference, Anaheim, CA, 2007.

[5] N. K. Singhal, L. H. Sahasrabuddhe, and B. Mukherjee, Provisioning of survivable multicast sessions against single link failures in optical WDM mesh networks, in J. Lightw. Technol., vol. 21, No. 11, pp. 2587-2594, 2003.

[6] F. Zhang, and W. D. Zhong, p-Cycle based tree protection of optical multicast traffic for combined link and node failure recovery in WDM mesh networks, in IEEE Commun. Lett., Vol. 13, No. 1, pp. 40-42, 2009.

[7] F. Zhang and W. D. Zhong, Performance evaluation of optical multicast protection approaches for combined node and link failure recovery, in $J$. Lightw Technol., Vol. 27, No. 18, pp. 4017-4025, 2009.

[8] A. Frikha, B. Cousin, and S. Lahoud, Extending Node Protection Concept of P-Cycles for an efficient Resource Utilization in Multicast Traffic, in proceedings of the 36th IEEE Conference on Local Computer Networks (LCN), pp. 175-178, Bonn, Germany, 2011.

[9] X. Wang, S. Wang, and L. Li, Protecting multicast sessions in WDM networks with sparse-splitting constraints, in ETRI Journal, vol. 29, No. 4, pp. 524-526, 2007.

[10] X. Wang, S. Wang, and L. Li, Provisioning of Survivable Multicast Sessions in Sparse Light Splitting WDM Networks, in proceedings of IEEE ICC, pp. 5286-5291, 2008.

[11] M. Ali, J.S. Deogun, Cost-effective implementation of multicasting in Wavelength-Routed Networks, in J. Lightw Technol., vol. 18, no. 12, pp. $1628-1638,2000$

[12] F. Zhou, M. Molnar, B. Cousin, Distance Priority Based Multicast Routing in WDM Networks Considering Sparse Light Splitting, in proceedings of IEEE ICCS, pp. 709-714, Guangzhou, 2008.

[13] S. De Maesschalck and al., Pan-European Optical Transport Networks: an Availability based Comparison, in Photonic Network Communications, vol. 5, no. 3, pp. 203-226, 2003.

[14] P. Batchelor et al.: Ultra High Capacity Optical Transmission Networks. in Final report of Action COST 239, 1999. 